\title{
Solutions for higher-order anisotropic elliptic-parabolic equations in time unbounded domains
}

\author{
Mykola Bokalo and Iryna Skira \\ Department of Differential Equations, Ivan Franko National University of Lviv, Ukraine
}

Received: 30 November 2016, Accepted: 23 October 2017

Published online: 26 March 2018.

\begin{abstract}
The problem without initial conditions or, in other words, the Fourier problem for anisotropic elliptic-parabolic equations with variable exponents of nonlinearity in time unbounded domains is considered in this paper. The existence and uniqueness solutions of the problem are proved with no conditions on the behavior of solutions and growth of input data at infinity. The estimates of these solutions are received. In addition, some properties of the weak solutions of the Fourier problem are considered. The conditions for existence of periodic solutions of the considered equations are set. Also the conditions for existence of Bohr almost periodic solutions and Stepanov almost periodic solutions of some equations are obtained.
\end{abstract}

Keywords: Fourier problem, elliptic-parabolic equation, periodic solution, Bohr almost periodic solutions, Stepanov almost periodic solutions.

\section{Introduction}

The problem without initial conditions or, in other words, the Fourier problem for evolution equations describes processes that started a long time ago and initial conditions do not affect on them in the actual time moment. Then the equations are considered for time variable beginning from $-\infty$. The Fourier problem for evolution equations has been widely used to study the models appearing in many fields of science such as economics, computer science, physics, ecology, cybernetics, etc. So these problems are examined in many papers; see, e.g., [1] - [11]. A lot of information about results concerning problems without initial conditions can be found in [10]. Note that the Fourier problem for linear and a plenty of nonlinear evolution equations are well-posed only if there are some restrictions on behavior of solutions and growth of input data as the time variable converges to $-\infty$ [1] - [7], [10]. But there are nonlinear parabolic equations for which the Fourier problem is uniquely solvable with no conditions at infinity [8] - [9], [11], [12].

Here we consider the higher-order anisotropic elliptic-parabolic equations with variable exponents of nonlinearity. These equations are defined on unbounded cylindrical domains which are the Cartesian products of bounded space domains and the whole time axis. For these equations we find the weak solutions, which defined for all values of the time axis (global solutions) and satisfied the boundary conditions. Since exponents of nonlinearity is variable then the weak solution of considered problems belong to the generalized Lebesgue and Sobolev spaces. More information on these spaces and its applications can be found in [13] - [18]. It is proved existence and uniqueness of weak solutions of considered problems with no conditions on their behavior and growth of coefficients and right sides of equations at infinity in this paper. Also the estimates of these solutions are received.

Note that from lack of initial conditions, there is a possibility to consider the conditions for the existence of periodic and almost periodic solutions. The theory of almost periodic functions was mainly treated and created by Bohr during 
1924-1926. For details about Bohr almost periodic functions, see [19]. Stepanov generalized the class of almost periodic functions in the sense of Bohr without using the hypothesis of continuity. More information on these functions can be found in [20]-[22].

In this paper we set the conditions for the existence of periodic solutions, Bohr almost periodic solutions and Stepanov almost periodic solutions. Similar results are in [12], but for the second order equation.

This paper consists of four parts: the first part is the introduction, in the second we say the formulation of the problem and main results, the third part encloses the auxiliary statements while the proofs of main results are in the fourth part.

\section{Formulation of the problem and main results}

Let $n$ be a natural number and $\mathbb{R}^{n}$ be the linear space consisting of ordered collections of real numbers $x=\left(x_{1}, \ldots, x_{n}\right)$ with norm $|x|:=\left(\left|x_{1}\right|^{2}+\ldots+\left.x_{n}\right|^{2}\right)^{1 / 2}$. Let $M$ be a subset of the set $\{0,1, \ldots, m\}$ such that $\{0, m\} \subset M$, where $m$ is some natural number. Denote by $N$ the number of $n$-dimensional multi-indexes $\alpha=\left(\alpha_{1}, \ldots, \alpha_{n}\right)$ (for each $i \in\{1, \ldots, n\}, \alpha_{i}$ is a nonnegative integer), the length $|\alpha|=\alpha_{1}+\ldots+\alpha_{n}$ of which is an element of the set $M$, and by $\mathbb{R}^{N}$ denote the linear space of ordered collections of $N$ real numbers $\xi=\left(\xi_{\hat{0}}, \ldots, \xi_{\alpha}, \ldots\right) \equiv\left(\xi_{\alpha}:|\alpha| \in M\right)$, components are numbered $n$-dimensional multi-indexes of $\alpha$, whose length $|\alpha|$ belongs to the set $M$, and ordered lexicographically (this mean, that $\alpha=\left(\alpha_{1}, \ldots, \alpha_{n}\right)$ precedes $\beta=\left(\beta_{1}, \ldots, \beta_{n}\right)$, when either $|\alpha|<|\beta|$, or $|\alpha|=|\beta|$ and $\alpha_{k}<\beta_{k}$, where $\left.k=\min \left\{j: \alpha_{j} \neq \beta_{j}\right\}\right)$. Hereafter $\widehat{0}$ is a multi-index, consisting of zeros. Put $|\xi|:=\left(\sum_{|\alpha| \in M}\left|\xi_{\alpha}\right|^{2}\right)^{\frac{1}{2}}$ for arbitrary $\xi \in \mathbb{R}^{N}$.

Let $\Omega$ be a bounded domain in $\mathbb{R}^{n}$ with piecewise smooth boundary $\Gamma:=\partial \Omega$. We denote by $v=\left(v_{1}, \ldots, v_{n}\right)$ the unit normal vector to pointing outward $\Gamma$. Set $Q:=\Omega \times \mathbb{R}, \Sigma:=\Gamma \times \mathbb{R}$ and $Q_{t_{1}, t_{2}}:=\Omega \times\left(t_{1}, t_{2}\right)$ for arbitrary real numbers $t_{1}$ and $t_{2}$ such that $t_{1}<t_{2}$.

Consider the problem of finding a function $u: \bar{Q} \rightarrow \mathbb{R}$ satisfying (in some sense) the equation

$$
(b(x) u)_{t}+\sum_{|\alpha| \in M}(-1)^{|\alpha|} D^{\alpha} a_{\alpha}(x, t, \delta u)=\sum_{|\alpha| \in M}(-1)^{|\alpha|} D^{\alpha} f_{\alpha}(x, t), \quad(x, t) \in Q
$$

and the boundary conditions

$$
\left.\frac{\partial^{j} u}{\partial v^{j}}\right|_{\Sigma}=0, \quad j=\overline{0, m-1}
$$

where $a_{\alpha}: Q \times \mathbb{R}^{N} \rightarrow \mathbb{R}, f_{\alpha}: Q \rightarrow \mathbb{R}(|\alpha| \in M)$ are given functions, $\delta u$ is the ordered collection of derivatives

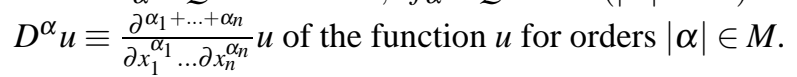

We should consider a weak solution of the problem (1), (2). For this, first define some functional spaces and classes of input data of the given problem.

Let $r \in L_{\infty}(\Omega)$ such that $r(x) \geq 1$ for almost each $x \in \Omega$. Suppose that either $G=\Omega$ or $G=\Omega \times S$, where $S$ is an interval in $\mathbb{R}$. Denote by $L_{r(\cdot)}(G)$ the generalized Lebesgue space consisting of the measurable functions $v: G \rightarrow \mathbb{R}$ such that $\rho_{G, r}(v)<\infty$, where $\rho_{G, r}(v):=\int_{G}|v(x)|^{r(x)} d x$ for $G=\Omega$, and $\rho_{G, r}(v):=\int_{G}|v(x, t)|^{r(x)} d x d t$ for $G=\Omega \times S$. The space is equipped with the norm $\|v\|_{L_{r(\cdot)}(G)}:=\inf \left\{\lambda>0 \mid \rho_{G, r}(v / \lambda) \leq 1\right\}$; it is a Banach space [11]. If $\underset{x \in \Omega}{\operatorname{ess}} \inf r(x)>1$, then the dual space $\left[L_{r(\cdot)}(G)\right]^{\prime}$ can be identified with $L_{r^{\prime}(\cdot)}(G)$, where $r^{\prime}$ is the function defined by the equality $\frac{1}{r(x)}+\frac{1}{r^{\prime}(x)}=1$ for almost each $x \in \Omega$. 
Let $G=\Omega \times S$, where $S$ is an unbounded open interval of $\mathbb{R}$ or $S=\mathbb{R}$. We denote by $L_{r(\cdot), \text { loc }}(\bar{G})$ the space of measurable functions $g: G \rightarrow \mathbb{R}$ such that the restriction of $g$ on $Q_{t_{1}, t_{2}}$ belongs to $L_{r(\cdot)}\left(Q_{t_{1}, t_{2}}\right)$ for each $t_{1}, t_{2} \in S$. It is complete locally convex linear space with respect to the family of seminorms

$\left\{\|\cdot\|_{L_{r(\cdot)}\left(Q_{t_{1}, t_{2}}\right)} \mid t_{1}, t_{2} \in S\right\}$. Therefore, a sequence $\left\{g_{m}\right\}$ is convergent strongly (resp., weakly) in $L_{r(\cdot), \text { loc }}(\bar{G})$ provided the sequences of restrictions $\left\{\left.g_{m}\right|_{Q_{t_{1}, t_{2}}}\right\}$ are convergent strongly (resp., weakly) in $L_{r(\cdot)}\left(Q_{t_{1}, t_{2}}\right)$ for all $t_{1}, t_{2} \in S$. Similarly we can define the space $L_{\infty, \text { loc }}(\bar{G})$.

We denote $W_{q}^{m}(\Omega):=\left\{v \in L_{q}(\Omega)\left|D^{\alpha} v \in L_{q}(\Omega) \forall \alpha,\right| \alpha \mid \leq m\right\}$, where $q \geq 1$ is any number. It is a Banach space with the norm $\|v\|_{W_{q}^{m}(\Omega)}:=\sum_{|\alpha| \leq m}\left\|D^{\alpha} v\right\|_{L_{q}(\Omega)}$ (see, for example, [23]). For each $q \geq 1$ and interval $S \subset \mathbb{R}$, let $W_{q}^{m, 0}(\Omega \times S):=\left\{h \in L_{q}(\Omega \times S)\left|D^{\alpha} h \in L_{q}(\Omega \times S) \forall \alpha,\right| \alpha \mid \leq m\right\}$. It is a Banach space with norm $\|h\|_{W_{q}^{m, 0}(\Omega \times S)}:=\sum_{|\alpha| \leq m}\left\|D^{\alpha} h\right\|_{L_{q}(\Omega \times S)}($ see, for example, [23]).

Assume that:

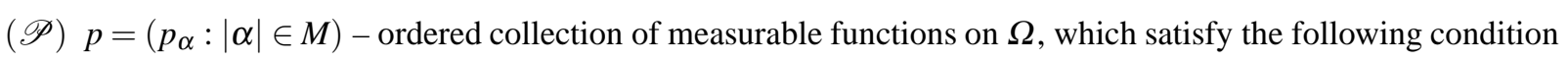

$$
2 \leq p_{\alpha}^{-}:=\underset{x \in \Omega}{\operatorname{essinf}} p_{\alpha}(x) \leq \underset{x \in \Omega}{\operatorname{ess} \sup } p_{\alpha}(x)=: p_{\alpha}^{+}<+\infty, \text { if }|\alpha| \in M, \text { while } p_{\widehat{0}}^{-}>2 .
$$

We also denote by $p^{\prime}=\left(p_{\alpha}{ }^{\prime}:|\alpha| \in M\right)$ an ordered set of functions such that $1 / p_{\alpha}(x)+1 / p_{\alpha}{ }^{\prime}(x)=1$ for almost each $x \in \Omega(|\alpha| \in M)$.

Let $W_{p(\cdot)}^{m}(\Omega):=\left\{v \in W_{2}^{m}(\Omega)\left|D^{\alpha} v \in L_{p_{\alpha}(\cdot)}(\Omega) \forall \alpha,\right| \alpha \mid \in M\right\}$. This space is a Banach space with the norm $\|v\|_{W_{p(\cdot)}^{m}(\Omega)}:=\sum_{|\alpha| \in M}\left\|D^{\alpha} v\right\|_{L_{p \alpha(\cdot)}(\Omega)}$. We define by $\stackrel{\circ}{W}_{p(\cdot)}^{m}(\Omega)$ closure of $C_{c}^{\infty}(\Omega)$ in $W_{p(\cdot)}^{m}(\Omega)\left(C_{c}^{\infty}(\Omega)\right.$ is the linear space of infinity differential functions defined on $\Omega$ with compact support). To simplify the presentation of the material we denote $\mathbb{V}_{p}:=\stackrel{\circ}{W_{p(\cdot)}^{m}}(\Omega)$.

For arbitrary $t_{1}, t_{2} \in \mathbb{R}\left(t_{1}<t_{2}\right)$ we define by $W_{p(\cdot)}^{m, 0}\left(Q_{t_{1}, t_{2}}\right)$ the subspace of the space $W_{2}^{m, 0}\left(Q_{t_{1}, t_{2}}\right)$, consisting of the functions $h$ such that $D^{\alpha} h \in L_{p_{\alpha}(\cdot)}\left(Q_{t_{1}, t_{2}}\right)$, if $|\alpha| \in M$, with the norm $\|h\|_{W_{p(\cdot)}^{m, 0}\left(Q_{t_{1}, t_{2}}\right)}:=\sum_{|\alpha| \in M}\left\|D^{\alpha} h\right\|_{L_{p \alpha(\cdot)}\left(Q_{t_{1}, t_{2}}\right)}$. We denote by $\stackrel{\circ}{W}_{p(\cdot),}^{m, 0}\left(Q_{t_{1}, t_{2}}\right)$ the subspace of the space $W_{p(\cdot)}^{m, 0},\left(Q_{t_{1}, t_{2}}\right)$, consisting of the functions $h$ such that $h(\cdot, t) \in \mathbb{V}_{p}$ for almost each $t \in\left[t_{1}, t_{2}\right]$.

Let $G=\Omega \times S$, where $S$ is an open unbounded interval of $\mathbb{R}$. Let us denote by $\stackrel{\circ}{W}_{p(\cdot), \text { loc }}^{m, 0}(\bar{G})$ the linear space of measurable functions such that their restrictions on $Q_{t_{1}, t_{2}}$ belong to $\stackrel{\circ}{W}_{p(\cdot)}^{m, 0}\left(Q_{t_{1}, t_{2}}\right)$ for all $t_{1}, t_{2} \in S$. It is complete locally convex linear space with respect to the family of seminorms $\left\{\|h\|_{W_{p(\cdot)}^{m, 0}\left(Q_{\left.t_{1}, t_{2}\right)}\right.}:=\sum_{|\alpha| \in M}\left\|D^{\alpha} h\right\|_{L_{p \alpha(\cdot)}\left(Q_{t_{1}, t_{2}}\right)} \mid t_{1}, t_{2} \in S\right\}$. Therefore, a sequence $\left\{h_{m}\right\}$ is convergent strongly (weakly) in $\stackrel{\circ}{\stackrel{W}{W}_{p(\cdot), \text { loc }}^{m, 0}}(\bar{G})$ provided the sequences of restrictions $\left\{\left.h_{m}\right|_{Q_{t_{1}, t_{2}}}\right\}$ are convergent strongly (weakly) in $\stackrel{\circ}{W}_{p(\cdot)}^{m, 0}\left(Q_{t_{1}, t_{2}}\right)$ for all $t_{1}, t_{2} \in S$.

The following assumption will be needed throughout the paper:

( $\mathscr{B}) \quad b: \Omega \rightarrow \mathbb{R}$ is a measurable bounded function, $0<b(x) \leq 1$ for $x \in \Omega_{0} \subset \Omega$ and $b(x)=0$ for $x \in \Omega \backslash \Omega_{0}$, where $\Omega_{0}$ is open set.

We need $0<b(x) \leq 1$ in the our proof. But if $\sup b(x)>1$ then we can divide equation by $\sup b(x)$ and use received $x \in \Omega$ $x \in \Omega$ 
results.

Let $\widetilde{b}(x)=b(x)$, if $x \in \Omega_{0}$, and $\widetilde{b}(x)=1$, if $x \in \Omega \backslash \Omega_{0}$. We denote by $H_{b}(\Omega)$ the linear space of functions of the form $w=\widetilde{b}^{-1 / 2} v$, where $v \in L_{2}(\Omega)$, with the seminorm $\|w\|_{H_{b}(\Omega)}=\left(\int_{\Omega} b(x)|w(x)|^{2} d x\right)^{1 / 2}$. It is easy to check that $H_{b}(\Omega)$ is the completion of $\mathbb{V}_{p}$ with respect to the seminorm $\|\cdot\|_{H_{b}(\Omega)}$ (see [7]).

Let $B$ be a linear space with a norm or a seminorm $\|\cdot\|_{B}$, S be a real interval. Let us denote by $C(S ; B)$ the space of functions $v: S \rightarrow B$ such that the restriction of $v$ on any interval $\left[t_{1}, t_{2}\right] \subset S$ belongs to $C\left(\left[t_{1}, t_{2}\right] ; B\right)$. It is complete locally convex linear space with respect to the family of seminorms $\left\{\|v\|_{C\left(\left[t_{1}, t_{2}\right] ; B\right)}:=\max _{t \in\left[t_{1}, t_{2}\right]}\|v(t)\|_{B} \mid t_{1}, t_{2} \in S\right\}$. A sequence $\left\{g_{m}\right\}$ is convergent in $C(S ; B)$ provided the sequences of restrictions $\left\{\left.g_{m}\right|_{\left[t_{1}, t_{2}\right]}\right\}$ are convergent in $C\left(\left[t_{1}, t_{2}\right] ; B\right)$ for each $t_{1}, t_{2} \in S$.

Set

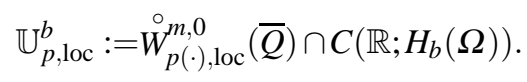

The space $\mathbb{U}_{p, \text { loc }}^{b}$ is a complete local convex linear space with respect to the family of seminorms

$$
\left\{\sum_{|\alpha| \in M}\left\|D^{\alpha} h\right\|_{L_{p \alpha(\cdot)}\left(Q_{t_{1}, t_{2}}\right)}+\|h\|_{\left.C\left(t_{1}, t_{2}\right] ; H_{b}(\Omega)\right)} \mid t_{1}, t_{2} \in \mathbb{R}\right\}
$$

A sequence $\left\{g_{m}\right\}$ is convergent in $\mathbb{U}_{p, \text { loc }}^{b}$ provided this sequence is convergent in $\stackrel{o}{W}_{p(\cdot), \text { loc }}^{m, 0}(\bar{Q})$ and $C\left(\mathbb{R} ; H_{b}(\Omega)\right)$.

Now let us denote by $\mathbb{A}_{p}$ the set of ordered collections of really valued functions $\left(a_{\alpha}:|\alpha| \in M\right)$, which are defined on $Q \times \mathbb{R}^{N}$ and satisfy the next three conditions:

$\left(\mathscr{A}_{1}\right)$ for each $\alpha(|\alpha| \in M)$ the function $a_{\alpha}(x, t, \xi),(x, t, \xi) \in Q \times \mathbb{R}^{N}$, is a Caratheodory (for almost all $(x, t) \in Q$ the function $a_{\alpha}(x, t, \cdot): \mathbb{R}^{N} \rightarrow \mathbb{R}$ is a continuous, and for all $\xi \in \mathbb{R}^{N}$ function $a_{\alpha}(\cdot, \cdot, \xi): Q \rightarrow \mathbb{R}$ is Lebesgue measurable), and $a_{\alpha}(x, t, 0)=0$ for almost all $(x, t) \in Q$;

$\left(\mathscr{A}_{2}\right)$ for each $\alpha(|\alpha| \in M)$, for almost all $(x, t) \in Q$ and for all $\xi \in \mathbb{R}^{N}$ we have

$$
\left|a_{\alpha}(x, t, \xi)\right| \leq h_{\alpha}(x, t) \sum_{|\beta| \in M}\left|\xi_{\beta}\right|^{p_{\beta}(x) / p_{\alpha}^{\prime}(x)}+g_{\alpha}(x, t),
$$

where $h_{\alpha} \in L_{\infty, \mathrm{loc}}(\bar{Q}), g_{\alpha} \in L_{p_{\alpha^{\prime}}(\cdot)}(\bar{Q})$;

$\left(\mathscr{A}_{3}\right)$ for almost all $(x, t) \in Q$ and for arbitrary $\xi, \eta \in \mathbb{R}^{N}$ the inequality

$$
\sum_{|\alpha| \in M}\left(a_{\alpha}(x, t, \xi)-a_{\alpha}(x, t, \eta)\right)\left(\xi_{\alpha}-\eta_{\alpha}\right) \geq K_{1} \sum_{|\alpha| \in M}\left|\xi_{\alpha}-\eta_{\alpha}\right|^{p_{\alpha}(x)}
$$

holds; here $K_{1}$ is a positive constant dependent on $\left(a_{\alpha}:|\alpha| \in M\right)$.

Also consider a subset $\mathbb{A}_{p}^{*}$ of set $\mathbb{A}_{p}$, consisting of collections of functions

$$
\left(a_{\alpha}(x, t, \xi) \equiv \widehat{a}_{\alpha}(x, t)\left|\xi_{\alpha}\right|^{p_{\alpha}(x)-2} \xi_{\alpha}:|\alpha| \in M\right),
$$


where, for all $\alpha,|\alpha| \in M, \widehat{a}_{\alpha} \in L_{\infty, \mathrm{loc}}(\bar{Q})$ and

$$
\widehat{a}_{\alpha}(x, t) \geq K_{2}>0 \quad \text { for almost all } \quad(x, t) \in Q,
$$

where $K_{2}$ is a positive constant, which may be dependent on $\left(\widehat{a}_{\alpha}:|\alpha| \in M\right)$.

The inclusion $\mathbb{A}_{p}^{*} \subset \mathbb{A}_{p}$ can be easily proved using the inequality

$$
\left(\left|s_{1}\right|^{q-2} s_{1}-\left|s_{2}\right|^{q-2} s_{2}\right)\left(s_{1}-s_{2}\right) \geq 2^{2-q}\left|s_{1}-s_{2}\right|^{q},
$$

where $q \geq 2, s_{1}, s_{2} \in \mathbb{R}$ are arbitrary.

Note that, if $\left(a_{\alpha}:|\alpha| \in M\right)$ belongs $\mathbb{A}_{p}^{*}$, then equation (1) has the form

$$
(b(x) u)_{t}+\sum_{|\alpha| \in M}(-1)^{|\alpha|} D^{\alpha}\left(\widehat{a}_{\alpha}(x, t)\left|D^{\alpha} u\right|^{p_{\alpha}(x)-2} D^{\alpha} u\right)=\sum_{|\alpha| \in M}(-1)^{|\alpha|} D^{\alpha} f_{\alpha}(x, t), \quad(x, t) \in Q .
$$

A partial case of the equation (4) (and, hence, the equation (1)) is the equation

$$
(b(x) u)_{t}+(-\Delta)^{m} u+|u|^{p_{0}(x)-2} u=f(x, t), \quad(x, t) \in Q .
$$

Let $\mathbb{F}_{p^{\prime}, \text { loc }}$ be the set consisting of ordered collections $\left(f_{\alpha}:|\alpha| \in M\right)$ of real valued functions defined on $Q$, and for each $\alpha(|\alpha| \in M)$ the function $f_{\alpha}$ belongs to the space $L_{p_{\alpha^{\prime}}(\cdot), \text { loc }}(\bar{Q})$.

Definition 1.Suppose that functions $b, p$ satisfy the conditions $(\mathscr{B}),(\mathscr{P})$, respectively, and $\left(a_{\alpha}:|\alpha| \in M\right) \in \mathbb{A}_{p},\left(f_{\alpha}\right.$ : $|\alpha| \in M) \in \mathbb{F}_{p^{\prime}, \text { loc. }}$. A function u is called a weak solution of (1), (2), provided $u \in \mathbb{U}_{p, l o c}^{b}$ and the following integral identity holds

$$
\iint_{Q}\left\{\sum_{|\alpha| \in M} a_{\alpha}(x, t, \delta u) D^{\alpha} v \varphi-b u v \varphi^{\prime}\right\} d x d t=\iint_{Q} \sum_{|\alpha| \in M} f_{\alpha} D^{\alpha} v \varphi d x d t
$$

for all $v \in \mathbb{V}_{p}, \varphi \in C_{c}^{1}(\mathbb{R})$.

The main results of our work are the next statements.

Theorem 1.Suppose that $b, p$ satisfy the conditions $(\mathscr{B})$, $(\mathscr{P})$, respectively, and $\left(a_{\alpha}:|\alpha| \in M\right) \in \mathbb{A}_{p},\left(f_{\alpha}:|\alpha| \in M\right) \in$ $\mathbb{F}_{p^{\prime}, \text { loc. }}$. Then there exists a unique weak solution of (1), (2), and the estimate

$$
\max _{t \in\left[t_{0}-R_{0}, t_{0}\right]} \int_{\Omega} b(x)|u(x, t)|^{2} d x+\int_{t_{0}-R_{0}}^{t_{0}} \int_{\Omega}\left(\sum_{|\alpha| \in M}\left|D^{\alpha} u(x, t)\right|^{p_{\alpha}(x)}\right) d x d t \leq C\left(R^{-2 /\left(p_{0}^{+}-2\right)}+\int_{t_{0}-R}^{t_{0}} \int_{\Omega} \sum_{|\alpha| \in M}\left|f_{\alpha}(x, t)\right|^{p_{\alpha}^{\prime}(x)} d x d t\right)
$$

holds for all $R, R_{0}, t_{0}$ such that $R_{0}>0, R \geq \max \left\{1,2 R_{0}\right\}, t_{0} \in \mathbb{R}$. Here $C>0$ is a constant which depends only on $K_{1}, p_{\alpha}^{-}$ $(|\alpha| \in M), \operatorname{mes}_{n} \Omega$.

Hereafter $\operatorname{mes}_{n} \Omega$ is the Lebesgue measure of $\Omega$.

Corollary 1. Under the assumptions of Theorem 1 , if $f_{\alpha} \in L_{p_{\alpha}^{\prime}(\cdot)}(Q)$ for each $\alpha,|\alpha| \in M$, then a weak solution of (1), (2) satisfies the estimate

$$
\sup _{t \in \mathbb{R}} \int_{\Omega} b(x)|u(x, t)|^{2} d x+\iint_{Q} \sum_{|\alpha| \in M}\left|D^{\alpha} u(x, t)\right|^{p_{\alpha}(x)} d x d t \leq C \iint_{Q} \sum_{|\alpha| \in M}\left|f_{\alpha}(x, t)\right|^{p_{\alpha}^{\prime}(x)} d x d t .
$$


Corollary 2. Under the assumptions of Theorem 1, if

$$
\sup _{\tau \in \mathbb{R}} \int_{\tau-1}^{\tau} \int_{\Omega} \sum_{|\alpha| \in M}\left|f_{\alpha}(x, t)\right|^{p_{\alpha}^{\prime}(x)} d x d t \leq C_{1}
$$

for some positive constant $C_{1}$, then a weak solution $u$ of (1), (2) satisfies the estimates

$$
\sup _{t \in \mathbb{R}} \int_{\Omega} b(x)|u(x, t)|^{2} d x \leq C_{2}, \quad \sup _{\tau \in \mathbb{R}} \int_{\tau-1}^{\tau} \int_{\Omega} \sum_{|\alpha| \in M}\left|D^{\alpha} u(x, t)\right|^{p_{\alpha}(x)} d x d t \leq C_{2},
$$

where $C_{2}>0$ is some positive constant being dependent on $K_{1}, p_{\alpha}^{-}(|\alpha| \in M)$ and $C_{1}$.

Corollary 3. Under the assumptions of Theorem 1, if

$$
\lim _{\tau \rightarrow-\infty(+\infty)} \int_{\tau-1}^{\tau} \int_{\Omega} \sum_{|\alpha| \in M}\left|f_{\alpha}(x, t)\right|^{p_{\alpha}^{\prime}(x)} d x d t=0
$$

then for a weak solution $u$ of problem (1), (2) the following equalities hold

$$
\lim _{t \rightarrow-\infty(+\infty)}\|u(\cdot, t)\|_{L_{2}(\Omega)}=0, \quad \lim _{\tau \rightarrow-\infty(+\infty)} \int_{\tau-1}^{\tau} \int_{\Omega} \sum_{|\alpha| \in M}\left|D^{\alpha} u(x, t)\right|^{p_{\alpha}(x)} d x d t=0
$$

Theorem 2.Under the assumptions of Theorem 1, if the functions $\left(a_{\alpha}:|\alpha| \in M\right),\left(f_{\alpha}:|\alpha| \in M\right)$ are time periodic with period $\sigma>0$, then the weak solution of (1), (2) is also $\sigma$-periodic in time.

A set $X \subset \mathbb{R}$ is called relatively dense, if there exists a positive $l$ such that for all $a \in \mathbb{R}$ interval $[a, a+l]$ contains at least one element of the set $X$, i.e., $X \cap[a, a+l] \neq \varnothing \forall a \in \mathbb{R}$.

Let $B$ be a linear space with a norm or a seminorm $\|\cdot\|_{B}$. A function $v \in C(\mathbb{R} ; B)$ is Borh almost periodic if for each $\varepsilon>0$ the set $\left\{\sigma \mid \sup _{t \in \mathbb{R}}\|v(\cdot, t+\sigma)-v(\cdot, t)\|_{B} \leq \varepsilon\right\}$ is relatively dense.

A function $f \in L_{p_{\alpha^{\prime}(\cdot), l o c}}(\bar{Q})$ is Stepanov almost periodic provided the set

$$
\left\{\sigma\left|\sup _{\tau \in \mathbb{R}} \int_{\tau-1}^{\tau} \int_{\Omega}\right| f_{\alpha}(x, t+\sigma)-\left.f_{\alpha}(x, t)\right|^{p^{\prime}(x)} d x d t \leq \varepsilon\right\}
$$

is relatively dense for each positive $\varepsilon(|\alpha| \in M)$.

We say that $w$ is Stepanov almost periodic as element $\stackrel{\circ}{W}_{p(\cdot), l o c}^{m, 0}(\bar{Q})$, if for each $\varepsilon>0$ the set

$$
\left\{\sigma \mid \sup _{\tau \in \mathbb{R}} \int_{\tau-1}^{\tau} \int_{\Omega}\left[\sum_{\mid \alpha \in M}\left|D^{\alpha} w(x, t+\sigma)-D^{\alpha} w(x, t)\right|^{p_{\alpha}(x)}\right] d x d t \leq \varepsilon\right\}
$$

is relatively dense. We refer to [4], [19] - [12] for the detailed information on the theory of almost periodic functions. 
Theorem 3.Under the assumptions of Theorem 1, let $\left(a_{\alpha}:|\alpha| \in M\right) \in A_{p}^{*}$ and for each $\alpha(|\alpha| \in M) \widehat{a}_{\alpha} \in C\left(\mathbb{R} ; L_{\infty}(\Omega)\right)$ is Borh almost periodic, $f_{\alpha} \in L_{p_{\alpha}^{\prime}(\cdot), l o c}(\bar{Q})$ is Stepanov almost periodic. Moreover, the set

$$
F_{\delta}:=\left\{\sigma\left|\sup _{\tau \in \mathbb{R}} \int_{\tau-1}^{\tau} \int_{\Omega} \sum_{\mid \alpha \in M}\right| f_{\alpha}(x, t+\sigma)-\left.f_{\alpha}(x, t)\right|^{p_{\alpha}^{\prime}(x)} d x d t \leq \delta, \max _{|\alpha| \in M} \sup _{t \in \mathbb{R}}\left\|a_{\alpha}(\cdot, t+\sigma)-a_{\alpha}(\cdot, t)\right\|_{L_{\infty}(\Omega)} \leq \delta\right\}
$$

is relatively dense for each $\delta>0$.

Then the (unique) weak solution of (4), (2) is Borh almost periodic as element $C\left(\mathbb{R} ; H_{b}(\Omega)\right)$ and Stepanov almost periodic as element $\stackrel{\circ}{W}_{p(\cdot), l o c}^{m, 0}(\bar{Q})$.

\section{Auxiliary statements}

The following statement is similarly to Lemma 1 in [24], and can be proved so as this lemma.

Lemma 1.Suppose that $b$, $p$ satisfy the conditions $(\mathscr{B}),(\mathscr{P})$, respectively. Given $t_{1}, t_{2} \in \mathbb{R}$ such that $t_{2}-t_{1} \geq 1$, we assume that a function $w \in \stackrel{\circ}{W_{p(\cdot)}^{m, 0}}\left(Q_{t_{1}, t_{2}}\right)$ satisfies the identity

$$
\int_{t_{1}}^{t_{2}} \int_{\Omega}\left\{\left(\sum_{|\alpha| \in M} g_{\alpha} D^{\alpha} v\right) \varphi-b w v \varphi^{\prime}\right\} d x d t=0, \quad v \in \mathbb{V}_{p}, \varphi \in C_{c}^{1}\left(t_{1}, t_{2}\right)
$$

for some functions $g_{\alpha} \in L_{p_{\alpha}^{\prime}(\cdot)}\left(Q_{t_{1}, t_{2}}\right)(|\alpha| \in M)$. Then $w \in C\left(\left[t_{1}, t_{2}\right] ; H_{b}(\Omega)\right)$ and the following equality

$$
\begin{array}{r}
\theta\left(\tau_{2}\right) \int_{\Omega} b(x) w\left(x, \tau_{2}\right) v(x) d x-\theta\left(\tau_{1}\right) \int_{\Omega} b(x) w\left(x, \tau_{1}\right) v(x) d x+\int_{\tau_{1}} \int_{\Omega}^{\tau_{2}}\left\{\left(\sum_{|\alpha| \in M} g_{\alpha} D^{\alpha} v\right) \theta-b w v \theta^{\prime}\right\} d x d t=0, \\
\frac{1}{2} \theta\left(\tau_{2}\right)\left\|w\left(\cdot, \tau_{2}\right)\right\|_{H_{b}(\Omega)}^{2}-\frac{1}{2} \theta\left(\tau_{1}\right)\left\|w\left(\cdot, \tau_{1}\right)\right\|_{H_{b}(\Omega)}^{2}-\frac{1}{2} \int_{\tau_{1}}^{\tau_{2}}\|w(\cdot, t)\|_{H_{b}(\Omega)}^{2} \theta^{\prime}(t) d t+\int_{\tau_{1}}^{\tau_{2}} \int_{\Omega}\left(\sum_{|\alpha| \in M} g_{\alpha} D^{\alpha} w\right) \theta d x d t=0
\end{array}
$$

holds for all $\theta \in C^{1}\left(\left[t_{1}, t_{2}\right]\right), v \in \mathbb{V}_{p}$ and $\tau_{1}, \tau_{2} \in\left[t_{1}, t_{2}\right]\left(\tau_{1}<\tau_{2}\right)$.

Lemma 2.Suppose that $b, p$ satisfy the conditions $(\mathscr{B}),(\mathscr{P})$, respectively, $\left(a_{\alpha}:|\alpha| \in M\right) \in \mathbb{A}_{p}$. Given $t_{1}, t_{2} \in \mathbb{R}$ such

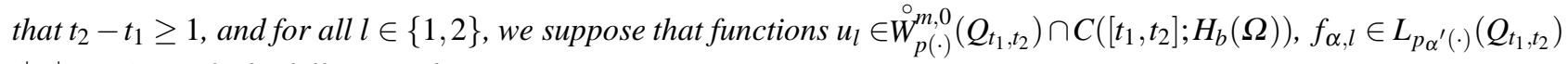
$(|\alpha| \in M)$ satisfy the following identities

$$
\int_{t_{1}}^{t_{2}} \int_{\Omega}\left\{\sum_{|\alpha| \in M} a_{\alpha}\left(x, t, \delta u_{l}\right) D^{\alpha} v \varphi-b u_{l} v \varphi^{\prime}\right\} d x d t=\int_{t_{1}}^{t_{2}} \int_{\Omega} \sum_{|\alpha| \in M} f_{\alpha, l} D^{\alpha} v \varphi d x d t
$$

for all $v \in \mathbb{V}_{p}$ and $\varphi \in C_{c}^{1}\left(t_{1}, t_{2}\right)$.

Then for each $R, R_{0}, t_{0}$ such, that $R_{0}>0, R \geq \max \left\{1,2 R_{0}\right\}, t_{1} \leq t_{0}-R<t_{0} \leq t_{2}$, the inequality

$$
\max _{t \in\left[t_{0}-R_{0}, t_{0}\right]} \int_{\Omega} b(x)\left|u_{1}(x, t)-u_{2}(x, t)\right|^{2} d x+\int_{t_{0}-R_{0}}^{t_{0}} \int_{\Omega}\left(\sum_{|\alpha| \in M}\left|D^{\alpha} u_{1}-D^{\alpha} u_{2}\right|^{p_{\alpha}(x)}\right) d x d t
$$




$$
\leq C\left\{R^{-2 /\left(p_{0}^{+}-2\right)}+\int_{t_{0}-R}^{t_{0}} \int_{|\alpha| \in M} \sum_{\mid \alpha, 1}\left|f_{\alpha, 2}\right|^{p^{\prime}(x)} d x d t\right\}
$$

holds (the constant $C$ in this lemma is the same as in Theorem 1).

Proof. Let $R, R_{0}, t_{0}$ be such as in the formulation of the lemma, and $\eta(t):=t-t_{0}+R, t \in \mathbb{R}$ (see also [26]). Given $v \in \mathbb{V} p$ and $\varphi \in C_{c}^{1}\left(t_{1}, t_{2}\right)$ we subtract the equality (11) when $l=1$ and the same equality when $l=2$. Then, for $(x, t) \in \Omega \times\left(t_{1}, t_{2}\right)$ putting

$$
\begin{gathered}
u_{12}(x, t):=u_{1}(x, t)-u_{2}(x, t), \\
f_{\alpha, 12}(x, t):=f_{\alpha, 1}(x, t)-f_{\alpha, 2}(x, t),|\alpha| \in M, \\
a_{\alpha, 12}(x, t):=a_{\alpha}\left(x, t, \delta u_{1}(x, t)\right)-a_{\alpha}\left(x, t, \delta u_{2}(x, t)\right),|\alpha| \in M,
\end{gathered}
$$

we obtain an equality

$$
\int_{t_{1}}^{t_{2}} \int_{\Omega}\left\{\left(\sum_{|\alpha| \in M} a_{\alpha, 12} D^{\alpha} v\right) \varphi-b u_{12} v \varphi^{\prime}\right\} d x d t=\int_{t_{1}}^{t_{2}} \int_{\Omega} \sum_{|\alpha| \in M} f_{\alpha, 12} D^{\alpha} v \varphi d x d t
$$

From this equality using Lemma 1 with $\tau_{1}=t_{0}-R, \tau_{2}=\tau \in\left(t_{0}-R, t_{0}\right], w=u_{12}, g_{\alpha}=a_{\alpha, 12}-f_{\alpha, 12}(|\alpha| \in M), \theta=\eta^{s}$, $s:=2 p_{0}^{-} /\left(p_{0}^{-}-2\right)$, we get the equality

$$
\begin{aligned}
& \eta^{s}(\tau) \int_{\Omega} b(x)\left|u_{12}(x, \tau)\right|^{2} d x+2 \int_{t_{0}-R \Omega}^{\tau} \int_{|\alpha| \in M} \sum_{\alpha, 12} D^{\alpha} u_{12} \eta^{s} d x d t \\
& =s \int_{t_{0}-R}^{\tau} \int_{\Omega} b\left|u_{12}\right|^{2} \eta^{s-1} d x d t+2 \int_{t_{0}-R}^{\tau} \int_{|\alpha| \in M} \sum_{\mid \alpha, 12} f_{\alpha,{ }^{\alpha}} u_{12} \eta^{s} d x d t .
\end{aligned}
$$

We make the corresponding estimates of the integrals of equality (14). From condition $\left(\mathscr{A}_{3}\right)$ we get

$$
\int_{t_{0}-R}^{\tau} \int_{\Omega} \sum_{|\alpha| \in M} a_{\alpha, 12} D^{\alpha} u_{12} \eta^{s} d x d t \geq K_{1} \int_{t_{0}-R}^{\tau} \int_{|\alpha| \in M} \sum_{|\alpha| \in{ }^{\alpha}}\left|u_{12}\right|^{p_{\alpha}(x)} \eta^{s} d x d t
$$

Further we need the following inequality:

$$
a c \leq \varepsilon|a|^{q}+\varepsilon^{-1 /(q-1)}|c|^{q^{\prime}}, \quad a, c \in \mathbb{R}, q>1,1 / q+1 / q^{\prime}=1, \varepsilon>0
$$

which is a corollary of the standard Young's inequality: $a c \leq|a|^{q} / q+|c|^{q^{\prime}} / q^{\prime}$.

Putting (for almost each $x \in \Omega) q=p_{0}(x) / 2, q^{\prime}=p_{0}(x) /\left(p_{0}(x)-2\right), a=b\left|u_{12}\right|^{2} \eta^{s / q}, c=\eta^{s / q^{\prime}-1}, \varepsilon=\varepsilon_{1} \in(0,1)$, under (16) we obtain

$$
\int_{t_{0}-R}^{\tau} \int_{\Omega} b\left|u_{12}\right|^{2} \eta^{s-1} d x d t \leq \varepsilon_{1} \int_{t_{0}-R}^{\tau} \int_{\Omega} b^{p_{0}(x) / 2}\left|u_{12}\right|^{p_{0}(x)} \eta^{s} d x d t+\varepsilon_{1}^{-2 /\left(p_{0}^{-}-2\right)} \int_{t_{0}-R}^{\tau} \int_{\Omega} \eta^{s-p_{0}(x) /\left(p_{0}(x)-2\right)} d x d t
$$

where $\varepsilon_{1} \in(0,1)$ is an arbitrary number. 
Again using inequality (16), we obtain

$$
\int_{t_{0}-R}^{\tau} \int_{\Omega} \sum_{|\alpha| \in M} f_{\alpha, 12} D^{\alpha} u_{12} \eta^{s} d x d t \leq \varepsilon_{2} \int_{t_{0}-R}^{\tau} \int_{\Omega} \sum_{|\alpha| \in M}\left|D^{\alpha} u_{12}\right|^{p_{\alpha}(x)} \eta^{s} d x d t+\int_{t_{0}-R}^{\tau} \int_{\Omega} \sum_{|\alpha| \in M} \varepsilon_{2}^{-1 /\left(p_{\alpha}^{-}-1\right)}\left|f_{\alpha, 12}\right|^{p_{\alpha}^{\prime}(x)} \eta^{s} d x d t
$$

where $\varepsilon_{2} \in(0,1)$ is an arbitrary number.

From (14) using (15), (17), (18), if $\varepsilon_{1}$ and $\varepsilon_{2}$ are sufficiently small, we obtain the following

$$
\begin{aligned}
& \eta^{s}(\tau) \int_{\Omega_{R}} b(x)\left|u_{12}(x, \tau)\right|^{2} d x+\int_{t_{0}-R}^{\tau} \int_{\Omega} \sum_{|\alpha| \in M}\left|D^{\alpha} u_{12}\right|^{p_{\alpha}(x)} \eta^{s} d x d t \\
& \leq C_{3}\left[\int_{t_{0}-R}^{\tau} \int_{\Omega} \eta^{s-p_{0}(x) /\left(p_{0}(x)-2\right)} d x d t+\int_{t_{0}-R}^{\tau} \int_{\Omega} \sum_{|\alpha| \in M}\left|f_{12}\right|^{p_{\alpha}^{\prime}(x)} \eta^{s} d x d t\right],
\end{aligned}
$$

where $C_{3}$ is a positive constant depending only on $K_{1}$ and $p_{\alpha}^{-}(|\alpha| \in M)$.

Since $0 \leq \eta(t) \leq R$, when $t \in\left[t_{0}-R, t_{0}\right]$, and $\eta(t) \geq R-R_{0}$, when $t \in\left[t_{0}-R_{0}, t_{0}\right]$, from (19) we obtain the inequality

$$
\begin{gathered}
\left(R-R_{0}\right)^{s} \int_{\Omega} b(x)\left|u_{12}(x, \tau)\right|^{2} d x+\left(R-R_{0}\right)^{s} \int_{t_{0}-R_{0}}^{\tau} \int_{\Omega} \sum_{|\alpha| \in M}\left|D^{\alpha} u_{12}\right|^{p_{\alpha}(x)} d x d t \\
\leq C_{3}\left[\int_{t_{0}-R}^{\tau} \int_{\Omega} R^{s-p_{0}(x) /\left(p_{0}(x)-2\right)} d x d t+R^{s} \int_{t_{0}-R}^{\tau} \int_{\Omega} \sum_{|\alpha| \in M}\left|f_{\alpha, 12}\right|^{p_{\alpha}^{\prime}(x)} d x d t\right] .
\end{gathered}
$$

We divide by $\left(R-R_{0}\right)^{s}$ the obtained inequality. Note that $R \geq \max \left\{1 ; 2 R_{0}\right\}$ (then, in particular, we have $R /\left(R-R_{0}\right)=$ $\left.1+R_{0} /\left(R-R_{0}\right) \leq 2\right)$. Using this inequality and $R^{-p_{0}(x) /\left(p_{0}(x)-2\right)} \leq R^{-p_{0}^{+} /\left(p_{0}^{+}-2\right)}$, for every $\tau \in\left[t_{0}-R_{0}, t_{0}\right]$ we obtain

$$
\begin{gathered}
\int_{\Omega_{R}} b(x)\left|u_{12}(x, \tau)\right|^{2} d x+\int_{t_{0}-R_{0} \Omega}^{\tau} \int_{|\alpha| \in M} \sum_{\mid}\left|D^{\alpha} u_{12}\right|^{p_{\alpha}(x)} d x d t \\
\leq C_{4}\left[R^{-p_{0}^{+} /\left(p_{0}^{+}-2\right)} \int_{t_{0}-R}^{\tau} \int_{\Omega} d x d t+\int_{t_{0}-R}^{\tau} \int_{\Omega} \sum_{|\alpha| \in M}\left|f_{12}\right|^{p_{\alpha}^{\prime}(x)} d x d t\right],
\end{gathered}
$$

where $C_{4}$ is a positive constant depending only on $K_{1}$ and $p_{\alpha}^{-}(|\alpha| \in M)$. Using (21) and the equality $\int_{t_{0}-R \Omega}^{t_{0}} \int d x d t=$ $R \cdot \operatorname{mes}_{n} \Omega$, we obtain (12).

\section{Proof of the main results}

\subsection{Proof of Theorem 1}

First we prove that there exists at most one weak solution of problem (1), (2). Assume the contrary. Let $u_{1}, u_{2}$ be (distinct) weak solutions of this problem. Using Lemma 2 , for arbitrary numbers $R, R_{0}, t_{0}$ with $R_{0}>0, R \geq \max \left\{1,2 R_{0}\right\}, t_{0} \in \mathbb{R}$ we 
get

$$
\int_{t_{0}-R_{0}}^{t_{0}} \int_{\Omega} \sum_{|\alpha| \in M}\left|D^{\alpha}\left(u_{1}-u_{2}\right)\right|^{p_{\alpha}(x)} d x d t \leq C R^{-2 /\left(p_{0}^{+}-2\right)}
$$

We fix arbitrary numbers $R_{0}>0$ and $t_{0} \in \mathbb{R}$ and take the limit as $R \rightarrow+\infty$ in (22). We receive that $u_{1}=u_{2}$ almost everywhere on $Q_{t_{0}-R_{0}, t_{0}}$. Since $R_{0}, t_{0}$ are arbitrary numbers, we obtain $u_{1}=u_{2}$ almost everywhere on $Q$. The obtained contradiction proves our statement.

Now we are turning to the proof of the existence of a weak solution of problem (1), (2). For each $m \in \mathbb{N}$ we consider an initial-boundary value problem for equation (1) in the domain $Q_{m}:=\Omega \times(-m,+\infty)$ with a homogeneous initial

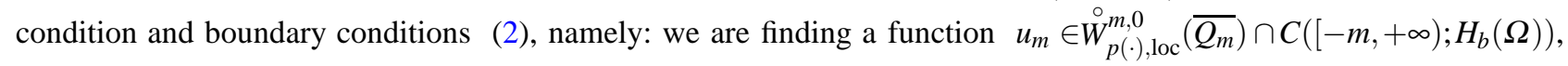
which satisfies the initial condition

$$
\left\|u_{m}(\cdot,-m)\right\|_{H_{b}(\Omega)}=0
$$

and the integral equality

$$
\iint_{Q_{m}}\left\{\sum_{|\alpha| \in M} a_{\alpha}\left(x, t, \delta u_{m}\right) D^{\alpha} v \varphi-b u_{m} v \varphi^{\prime}\right\} d x d t=\iint_{Q_{m}} \sum_{|\alpha| \in M} f_{\alpha, m} D^{\alpha} v \varphi d x d t \quad v \in \mathbb{V}_{p}, \varphi \in C_{c}^{1}(-m,+\infty),
$$

where $f_{\alpha, m}(x, t):=f_{\alpha}(x, t)$, if $(x, t) \in Q_{m}$, and $f_{\alpha, m}(x, t):=0$, if $(x, t) \in Q \backslash Q_{m}$. The existence and uniqueness of the function $u_{m}$ is proved in [27] (see also [7] and [24]). We extend $u_{m}$ on $Q$ by zero and this extension is denoted by $u_{m}$ again. Further we prove that the sequence $\left\{u_{m}\right\}$ converges in $\mathbb{U}_{p, l o c}^{b}$ to a weak solution of problem (1), (2). Indeed, note that for each $m \in \mathbb{N}$ the function $u_{m}$ is a weak solution of the problem which differs from problem (1), (2) by $f_{\alpha, m}$ instead of $f_{\alpha}$ for all $\alpha,|\alpha| \in M$. Using Lemma 2 for each natural numbers $m$ and $k$ we have

$$
\begin{gathered}
\max _{t \in\left[t_{0}, t_{0}-R_{0}\right]} \int_{\Omega} b(x)\left|u_{m}(x, t)-u_{k}(x, t)\right|^{2} d x+\int_{t_{0}-R_{0}}^{t_{0}} \int_{\Omega}\left(\sum_{|\alpha| \in M}\left|D^{\alpha} u_{m}-D^{\alpha} u_{k}\right|^{p_{\alpha}(x)}\right) d x d t \\
\leq C\left\{R^{-2 /\left(p_{0}^{+}-2\right)}+\int_{t_{0}-R}^{t_{0}} \int_{\Omega} \sum_{|\alpha| \in M}\left|f_{\alpha, m}(x, t)-f_{\alpha, k}(x, t)\right|^{p \alpha^{\prime}(x)} d x d t\right\},
\end{gathered}
$$

where $t_{0}, R_{0}, R$ are arbitrary numbers such that $t_{0} \in \mathbb{R}, R_{0} \geq 0, R \geq \max \left\{1,2 R_{0}\right\}$.

We show that for fixed $t_{0}$ and $R_{0}$ the left side of inequality (24) converges to zero when $m, k \rightarrow+\infty$. Let $\varepsilon>0$ be an arbitrary small number. Choose $R$ so big that

$$
C R^{-2 /\left(p_{0}^{+}-2\right)}<\varepsilon
$$

This is possible as $p_{0}^{+}-2>0$. By (25) for arbitrary $m, k \in \mathbb{N}$ such that $\max \{-m,-k\} \leq t_{0}-R$, we have $f_{\alpha, m}=f_{\alpha, k}$ $(|\alpha| \in M)$ almost everywhere on $\Omega \times\left(t_{0}-R, t_{0}\right)$ and the right side of inequality (24) is less than $\varepsilon$. This implies that the restriction of the terms of the sequence $\left\{u_{m}\right\}$ on $Q_{t_{0}-R_{0}, t_{0}}$ is a Cauchy sequence in $\stackrel{\circ}{W}_{p(\cdot)}^{m, 0}\left(Q_{t_{0}-R_{0}, t_{0}}\right) \cap C\left(\left[t_{0}-R_{0}, t_{0}\right] ; H_{b}(\Omega)\right)$. Therefore, since $t_{0}$ and $R_{0}$ are arbitrary, there exists a function $u \in \mathbb{U}_{p, \text { loc }}^{b}$ such that $u_{m} \rightarrow u$ in $\mathbb{U}_{p, \text { loc }}^{b}$. Note that in (23) the integration on $Q_{m}$ can be replaced by integration on $Q$ and take the limit of this equality for $m \rightarrow \infty$. So we get

$$
\iint_{Q}\left\{\sum _ { | \alpha | \in M } a _ { \alpha } ( x , t , \delta u _ { m } ) D ^ { \alpha } v \varphi d x d t \rightarrow \int \int _ { Q } \left\{\sum_{|\alpha| \in M} a_{\alpha}(x, t, \delta u) D^{\alpha} v \varphi d x d t\right.\right.
$$


(this fact implies from lemma 2.2 in [25]) and as a result we obtain (5) for all $v \in \mathbb{V}_{p}$ and $\varphi \in C_{c}^{1}(\mathbb{R})$. It means that the function $u$ is a weak solution of problem (1),(2). Estimate (6) directly follows from Lemma 2 putting $u_{1}=u, u_{2}=$ $0, f_{\alpha, 1}=f_{\alpha}, f_{\alpha, 2}=0(|\alpha| \in M)$.

\subsection{Proof of Corollaries 1-3}

These statements follow from estimate (6).

\subsection{Proof of Theorem 2}

Let $u$ is a weak solution of problem (1), (2). Put $u^{(\mu)}(x, t):=u(x, t+\mu), f_{\alpha}^{(\mu)}(x, t):=f_{\alpha}(x, t+\mu), a_{\alpha}^{(\mu)}(x, t, \xi):=a_{\alpha}(x, t+$ $\mu, \xi),(x, t) \in Q, \xi \in \mathbb{R}^{N}$, where $\mu \in \mathbb{R}$. Replace variable $t$ by $t+\mu$ ( $\mu \in \mathbb{R}$ is arbitrary) in (5). As a result, we obtain an identity

$$
\iint_{Q}\left\{\sum_{|\alpha| \in M} a_{\alpha}^{(\mu)}\left(x, t, \delta u^{(\mu)}\right) D^{\alpha} v \varphi-b u^{(\mu)} v \varphi^{\prime}\right\} d x d t=\iint_{Q} \sum_{|\alpha| \in M} f_{\alpha}^{(\mu)} D^{\alpha} v \varphi d x d t, \quad v \in \mathbb{V}_{p}, \varphi \in C_{c}^{1}(\mathbb{R}) .
$$

Rewrite this equality in the form

$$
\begin{aligned}
\iint_{Q}\left\{\sum_{|\alpha| \in M} a_{\alpha}^{(0)}\left(x, t, \delta u^{(\mu)}\right) D^{\alpha} v \varphi-b u^{(\mu)} v \varphi^{\prime}\right\} d x d t & =\iint_{Q} \sum_{|\alpha| \in M}\left\{a_{\alpha}^{(0)}\left(x, t, \delta u^{(\mu)}\right)-a_{\alpha}^{(\mu)}\left(x, t, \delta u^{(\mu)}\right)\right\} D^{\alpha} v \varphi d x d t \\
& +\iint_{Q} \sum_{|\alpha| \in M} f_{\alpha}^{(\mu)} D^{\alpha} v \varphi d x d t, \quad v \in \mathbb{V}_{p}, \varphi \in C_{c}^{1}(\mathbb{R}) .
\end{aligned}
$$

From (26), putting $\mu=\sigma$ and using periodicity of the functions $a_{\alpha}$ and $f_{\alpha}(|\alpha| \in M)$, we obtain that the function $u^{(\sigma)}$ is a weak solution of problem (1), (2). Taking this into consideration and the fact of uniqueness of a weak solution of the problem (1), (2), we get $u^{(0)}=u^{(\sigma)}$ almost everywhere on $Q$. Therefore the statement of Theorem 2 is correct.

\subsection{Proof of Theorem 3}

For every $\mu \in \mathbb{R}$ we put

$$
a_{\alpha}^{(\mu)}[w](x, t):=\hat{a}_{\alpha}(x, t+\mu)\left|D^{\alpha} w(x, t)\right|^{p_{\alpha}(x)-2} D^{\alpha} w(x, t),(x, t) \in Q(|\alpha| \in M) .
$$

Similarly as in the proof of Theorem 2 (see (26)), we get the identity

$$
\iint_{Q}\left\{\sum_{|\alpha| \in M} a_{\alpha}^{(0)}\left[u^{(\mu)}\right] D^{\alpha} v \varphi-b u^{(\mu)} v \varphi^{\prime}\right\} d x d t=\iint_{Q} \sum_{|\alpha| \in M}\left\{a_{\alpha}^{(0)}\left[u^{(\mu)}\right]-a_{\alpha}^{(\mu)}\left[u^{(\mu)}\right]+f_{\alpha}^{(\mu)}\right\} D^{\alpha} v \varphi d x d t, v \in \mathbb{V}_{p}, \varphi \in C_{c}^{1}(\mathbb{R}) .
$$

Let $\delta_{*}:=\min \left\{1 ; K_{1} / 2\right\}>0$ and $\sigma \in F_{\delta_{*}}$, where $F_{\delta}$ is defined over theorem. Consider the identity (27) first for $\mu=0$, and then for $\mu=\sigma \neq 0$. Then, using Lemma 2 with $u_{1}=u^{(0)}, u_{2}=u^{(\sigma)}, a_{\alpha}(x, t, \xi)=\widehat{a}_{\alpha}(x, t)\left|\xi_{\alpha}\right|^{p_{\alpha}(x)-2} \xi_{\alpha}(|\alpha| \in M), f_{\alpha, 1}=$ $f_{\alpha}^{(0)}, f_{\alpha, 2}=a_{\alpha}^{(0)}\left[u^{(\sigma)}\right]-a_{\alpha}^{(\sigma)}\left[u^{(\sigma)}\right]+f_{\alpha}^{(\sigma)}(|\alpha| \in M), t_{0}=\tau \in \mathbb{R}$ is arbitrary, $R_{0}=1, R=l \in \mathbb{N}$ and $l \geq 2$, we get

$$
\max _{t \in[\tau-1, \tau]} \int_{\Omega} b(x)\left|u^{(\sigma)}(x, t)-u^{(0)}(x, t)\right|^{2} d x+\int_{\tau-1}^{\tau} \int_{\Omega}\left(\sum_{|\alpha| \in M}\left|D^{\alpha} u^{(\sigma)}-D^{\alpha} u^{(0)}\right|^{p_{\alpha}(x)}\right) d x d t
$$




$$
\leq C\left\{l^{-2 /\left(p_{0}^{+}-2\right)}+\int_{\tau-l}^{\tau} \int_{\Omega} \sum_{|\alpha| \in M}|| a_{\alpha}^{(0)}\left[u^{\sigma}\right]-a_{\alpha}^{(\sigma)}\left[u^{\sigma}\right]|+| f_{\alpha}^{(\sigma)}-f_{\alpha}^{(0)}||^{p_{\alpha}^{\prime}(x)} d x d t\right\} .
$$

By the inequality: $(a+c)^{v} \leq 2^{v-1}\left(a^{v}+c^{v}\right), \quad a \geq 0, c \geq 0, v \geq 1$, we have

$$
\begin{gathered}
\int_{\tau-l}^{\tau} \int_{\Omega} \sum_{|\alpha| \in M}|| a_{\alpha}^{(0)}\left[u^{(\sigma)}\right]-a_{\alpha}^{(\sigma)}\left[u^{(\sigma)}\right]|+| f_{\alpha}^{(\sigma)}-f_{\alpha}^{(0)}||^{p_{\alpha}(x)} d x d t \\
\leq \sum_{|\alpha| \in M} 2^{1 /\left(p_{\alpha}^{-}-1\right)} \int_{\tau-l}^{\tau} \int_{\Omega}\left(\left|a_{\alpha}^{(\sigma)}\left[u^{\sigma}\right]-a_{\alpha}^{(0)}\left[u^{\sigma}\right]\right|^{p_{\alpha}^{\prime}(x)}+\left|f_{\alpha}^{(\sigma)}-f_{\alpha}^{(0)}\right|^{p_{\alpha}^{\prime}(x)}\right) d x d t .
\end{gathered}
$$

For $\alpha,|\alpha| \in M$, we have

$$
\begin{aligned}
\int_{\tau-l}^{\tau} \int_{\Omega}\left|a_{\alpha}^{(\sigma)}\left[u^{\sigma}\right]-a_{\alpha}^{(0)}\left[u^{\sigma}\right]\right|^{p_{\alpha}^{\prime}(x)} d x d t & =\int_{\tau-l}^{\tau} \int_{\Omega}\left|\widehat{a}_{\alpha}(x, t+\sigma)-\widehat{a}_{\alpha}(x, t)\right|^{p_{\alpha}^{\prime}(x)}\left|D^{\alpha} u^{(\sigma)}\right|^{p_{\alpha}(x)} d x d t \\
& \leq\left(\sup _{t \in \mathbb{R}}|| \hat{a}_{\alpha}(\cdot, t+\sigma)-\hat{a}_{\alpha}(\cdot, t) \|_{L_{\infty}(\Omega)}\right)^{\left(p_{\alpha}^{+}\right)^{\prime}} \int_{\tau-l}^{\tau} \int_{\Omega}\left|D^{\alpha} u^{(\sigma)}\right| p^{p_{\alpha}(x)} d x d t .
\end{aligned}
$$

Since $u^{(\sigma)}$ is a weak solution of problem, which differs from (1), (2) only in that we have $a_{\alpha}(x, t+\sigma, \xi)$ instead $a_{\alpha}(x, t, \xi)$ $(|\alpha| \in M)$ and $f_{\alpha}^{(\sigma)}$ instead $f_{\alpha}$, than $u^{(\sigma)}$ satisfies estimate (6) with replace $u$ on $u^{(\sigma)}$ and $f_{\alpha}$ on $f_{\alpha}^{(\sigma)}(|\alpha| \in M)$. From this estimate, putting $t_{0}=\tau, R_{0}=l, R=2 l$ we receive

$$
\sum_{|\alpha| \in M_{\tau-l}} \int_{\Omega}^{\tau} \int_{\Omega}\left|D^{\alpha} u^{(\sigma)}\right|^{p_{\alpha}(x)} d x d t \leq C_{4}\left\{(2 l)^{-2 /\left(p_{0}^{+}-2\right)}+\int_{\tau-2 l}^{\tau} \int_{\Omega} \sum_{|\alpha| \in M}\left|f_{\alpha}^{(\sigma)}\right|^{p_{\alpha}(x)} d x d t\right\}
$$

Then from (28), using (29) - (31), we receive

$$
\begin{aligned}
& \int_{\Omega} b(x)\left|u^{(\sigma)}(x, \tau)-u^{(0)}(x, \tau)\right|^{2} d x+\int_{\tau-1}^{\tau} \int_{\Omega}\left(\sum_{|\alpha| \in M}\left|D^{\alpha} u^{(\sigma)}-D^{\alpha} u^{(0)}\right|^{p_{\alpha}(x)}\right) d x d t \\
& \leq C_{5}\left\{l^{-2 /\left(p_{0}^{+}-2\right)}+\sum_{k=1}^{l} \int_{\tau-k}^{\tau-k+1} \int_{\Omega} \sum_{|\alpha| \in M}\left(\left|f_{\alpha}^{(\sigma)}-f_{\alpha}^{(0)}\right|^{p_{\alpha}^{\prime}(x)} d x d t\right.\right. \\
& \left.+\max _{|\alpha| \in M}\left(\sup _{t \in \mathbb{R}}|| a_{\alpha}^{(\sigma)}(\cdot, t)-a_{\alpha}(\cdot, t) \|_{L_{\infty}(\Omega)}\right)^{\left(p_{\alpha}^{+}\right)^{\prime}}\left[l^{-2 /\left(p_{0}^{+}-2\right)}+\sum_{k=1}^{2 l} \int_{\tau-k}^{\tau-k+1} \int_{\Omega} \sum_{|\alpha| \in M}\left|f_{\alpha}^{(\sigma)}\right|^{p_{\alpha}(x)} d x d t\right]\right\},
\end{aligned}
$$

where $C_{5}>0$ is some positive constant independent of $\tau, \sigma$ and $l$.

Since for each $\alpha$, such that $|\alpha| \in M, f_{\alpha}$ are Stepanov almost periodic as an element $L_{p_{\alpha}^{\prime}(\cdot), \text { loc }}(\bar{Q})$, the estimate

$$
\sup _{\tau \in \mathbb{R}} \int_{\tau}^{\tau-1} \int_{\Omega} \sum_{|\alpha| \in M}\left|f_{\alpha}^{(\sigma)}\right|^{p^{\prime}(x)} d x d t \leq C_{6}
$$


holds; here $C_{6}>0$ is some constant independent of $\tau, \sigma$ and $l$.

Let $\varepsilon>0$ be an arbitrary small fixed number. We claim that the set

$$
\begin{gathered}
U_{\varepsilon}:=\left\{\sigma \in \mathbb{R}\left|\sup _{t \in \mathbb{R}} \int_{\Omega} b(x)\right| u(x, t+\sigma)-\left.u(x, t)\right|^{2} d x \leq \varepsilon,\right. \\
\left.\sup _{t \in \mathbb{R}_{\tau-1}} \int_{\Omega}^{\tau}\left[\sum_{|\alpha| \in M}\left|D^{\alpha} u(x, t+\sigma)-D^{\alpha} u(x, t)\right|^{p_{\alpha}(x)}\right] d x d t \leq \varepsilon\right\}
\end{gathered}
$$

contains a set $F_{\delta}$ for some $\delta \in\left(0, \delta_{*}\right]$. Indeed, choose and fix big enough $l \in \mathbb{N}(l \geq 2)$ satisfying the following inequality

$$
C_{5} l^{-2 /\left(p_{0}^{+}-2\right)} \leq \varepsilon / 2
$$

Then take $\delta \in\left(0, \delta_{*}\right]$ such that the following inequality remains true

$$
C_{5}\left(l \delta+\max _{|\alpha| \in M} \delta^{\left(p_{\alpha}^{+}\right)^{\prime}}\left(l^{-2 /\left(p_{0}^{+}-2\right)}+2 l C_{6}\right)\right) \leq \varepsilon / 2 .
$$

Therefore, if $\sigma \in F_{\delta}$, then the right side of the inequality (32) is less than or equal to $\varepsilon$. From this, we have that $F_{\delta} \subset U_{\varepsilon}$ and the set $U_{\varepsilon}$ is relative density. The fact we had to prove.

\section{Competing interests}

The authors declare that they have no competing interests.

\section{Authors' contributions}

All authors have contributed to all parts of the article. All authors read and approved the final manuscript.

\section{References}

[1] A. N. Tihonov, Uniqueness theorems for the heat equation, Matem. Sbornik, 2, 510-516 (1935).

[2] J.-L. Lions, Quelques méthodes de résolution des problémes aux limites non linéaires, Dunod Gauthier-Villars, Paris, (1969).

[3] O. A. Oleinik, G. A. Iosifjan, Analog of Saint-Venant's principle and uniqueness of solutions of the boundary problems in unbounded domain for parabolic equations, Usp. Mat. Nauk., 31(6), 142-166 (1976).

[4] A. A. Pankov, Bounded and almost periodic solutions of nonlinear operator differential equations, Kluwer, Dordrecht, (1990).

[5] N. M. Bokalo, Problem without initial conditions for some classes of nonlinear parabolic equations, J. Sov. Math., 51(3), 22912322 (1990).

[6] R. E. Showalter, Singular nonlinear evolution equations, Rocky Mountain J. Math., 10(3), 499-507 (1980).

[7] R. E. Showalter, Monotone operators in Banach space and nonlinear partial differential equations, Amer. Math. Soc., Providence, 49, (1997).

[8] M. M. Bokalo, V. M Sikorskyy., About properties of solutions of problem without initial conditions for equations generalized politropic filtration equation, Visnyk of the Lviv University. Serija meh.-mat., 51, 85-98 (1998).

[9] M. M. Bokalo, I. B. Pauchok, On the well-posedness of the Fourier problem for higher-order nonlinear parabolic equations with variable exponents of nonlinearity, Mat. Stud., 26(1), 25-48 (2006). 
[10] Mykola Bokalo, Alfredo Lorenzi, Linear evolution first-order problems without initial conditions, Milan Journal of Mathematics, 77, 437-494 (2009).

[11] Mykola Bokalo, Dynamical problems without initial conditions for elliptic-parabolic equations in spatial unbounded domains, Electronic Journal of Differential Equations, 178, 1-24 (2010).

[12] M. M. Bokalo, Almost periodic solutions of anisotropic elliptic-parabolic equations with variable exponents of nonlinearity, Electronic Journal of Differential Equations, 169, 1-13 (2014).

[13] O. Kováčik, J. Rákosníc, On spaces $L^{p(x)}$ and $W^{k, p(x)}$, Czechoslovak Mathematical Journal, 41(116), 592-618 (1991).

[14] Y. Fu, N. Pan, Existence of solutions for nonlinear parabolic problem with $p(x)$-growth, Journal of Mathematical Analysis and Applications, 362, 313-326 (2010).

[15] Y. Alkhutov, S. Antontsev, V. Zhikov, Parabolic equations with variable order of nonlinearity, Collection of works of Institute of Mathematics NAS of Ukraine, 6, 23-50 (2009).

[16] R. A. Mashiyev, O. M. Buhrii, Existence of solutions of the parabolic variational inequality with variable exponent of nonlinearity, Journal of Mathematical Analysis and Applications, 377, 450-463 (2011).

[17] L. Diening, P. Harjulehto, P. Hästö, M. Rǔžička, Lebesgue and Sobolev spaces with variable exponents, Lecture Notes in Mathematics, 2017. Springer, Heidelberg, (2011).

[18] S. Antontsev, S. Shmarev, Evolution PDEs with nonstandard growth conditions. Existence, uniqueness, localization, blow-up, Atlantis Studies in Differential Equations, 4. Atlantis Press, Paris, (2015).

[19] G. Borh, Almost periodic functions, M.: (1934).

[20] B. M. Levitan, V. V. Zhikov, Almost periodic functions and differential equations, Izd-vo Mosk. un-ta., Moscow, (1978).

[21] Z. Hu, Boundeness and Stepanov's almost periodicity of solutions, Electronic Journal of Differential Equations, 35, 1-7 (2005).

[22] Md. Maqbul, Almost periodic solutions of neutral functional differential equations with Stepanov-almost periodic terms. Electronic Journal of Differential Equations, 72, 1-9 (2011).

[23] L. C. Evans, Partial differential equations, Graduate Studies in Mathematics, Amer. Math. Soc., Providence, 19 (2010).

[24] M. M. Bokalo, O. M. Buhrii, R. A. Mashiev, Unique solvability of initial boundary value problems for anisotropic elliptic-parabolic equations wiyh variable exponents of nonlinearity, J. Nonlinear Evol. Edu. Appl., 2013(6), 67-87 (2013).

[25] H. Gayevskyy, K. Greger, K. Zaharias, Nonlinear operator equations and operator differential equations, Mir, Moscow, (1978).

[26] F. Bernis, Elliptic and parabolic semilinear problems without conditions at infinity, Arch. Ration. Mech. and Anal., 106(3), 217241 (1989).

[27] M. M. Bokalo, Mixed problem for higher-order anisotropic elliptic-parabolic equations with variable exponents of nonlinearity, Visnyk of the Lviv University. Serija meh.-mat., 78, 14-26 (2013). 